\title{
Dually flat structures induced from monotone metrics on a two-level quantum state space
}

\author{
Akio Fujiwara ${ }^{\mathrm{a}} \mathbb{D}$ \\ Department of Mathematics, Osaka University, Toyonaka, Osaka 560-0043, Japan
}

Received: 24 June 2020 / Accepted: 22 October 2020 / Published online: 27 October 2020

(C) The Author(s) 2020

\begin{abstract}
The notion of dually flatness is of central importance in information geometry. Nevertheless, little is known about dually flat structures on quantum statistical manifolds except that the Bogoliubov metric admits a global dually flat structure on a quantum state space $\mathcal{S}\left(\mathbb{C}^{d}\right)$ for any $d \geq 2$. In this paper, we show that every monotone metric on a two-level quantum state space $\mathcal{S}\left(\mathbb{C}^{2}\right)$ admits a local dually flat structure.
\end{abstract}

\section{Introduction}

A Riemannian metric $g$ on a flat manifold $(M, \nabla)$ is called a Hessian metric if there is a function $\psi$ on $M$ such that $g=\nabla d \psi$, or equivalently,

$$
g_{i j}=\frac{\partial^{2} \psi}{\partial \theta^{i} \partial \theta^{j}},
$$

where $\left(\theta^{i}\right)$ is a $\nabla$-affine local coordinate system [1]. It is well known that a metric $g$ on a flat manifold $(M, \nabla)$ is of Hessian type if and only if it satisfies the Codazzi equation

$$
\left(\nabla_{X} g\right)(Y, Z)=\left(\nabla_{Y} g\right)(X, Z) .
$$

Since $\nabla$ is assumed to be a flat connection, the integrability condition (2) is equivalent to saying that the dual affine connection $\nabla^{*}$ defined by

$$
X g(Y, Z)=g\left(\nabla_{X} Y, Z\right)+g\left(Y, \nabla_{X}^{*} Z\right)
$$

is torsion free. Thus, $(g, \nabla)$ is a Hessian structure if and only if $\left(g, \nabla, \nabla^{*}\right)$ is a (local) dually flat structure $[2,3]$.

As mentioned above, it is straightforward to verify whether a Riemannian metric $g$ on a flat manifold $(M, \nabla)$ is of Hessian type. In this paper, we are interested in a slightly different problem. Given a Riemannian manifold $(M, g)$, determine whether there is an affine connection $\nabla$ that makes $g$ of Hessian type. This is equivalent to asking whether there is a local coordinate system $\left(\theta^{i}\right)$ and a function $\psi$ that satisfy (1). If this is the case, we say that the Riemannian manifold $(M, g)$ admits a Hessian structure.

\footnotetext{
a e-mail: fujiwara@math.sci.osaka-u.ac.jp (corresponding author)
} 
This problem was raised in [2, p. 102] and was recalled in [3,4]. Determining whether a manifold admits a global dually flat structure was studied in [5]. For a local structure, a clarification was made by Bryant [6] and Amari and Armstrong [7], who proved the following

Proposition 1 If $\operatorname{dim} M=2$ and $g$ is analytic, a Riemannian manifold $(M, g)$ admits a Hessian structure. If $\operatorname{dim} M \geq 3$, a Riemannian manifold $(M, g)$ does not always admit a Hessian structure.

The idea behind the proof of Proposition 1 is simple. Letting $n=\operatorname{dim} M$, the left-hand side of (1) consists of $\frac{1}{2} n(n+1)$ functions $g_{i j}$, while the right-hand side of (1) depends only on $(n+1)$ functions $\theta^{1}, \ldots, \theta^{n}$, and $\psi$. Thus, if $n>2$, not every metric $g$ can be represented in the form (1). Note that Amari and Armstrong [7] further discussed a curvature obstruction to the existence of Hessian metrics when $\operatorname{dim} M \geq 4$.

Turning our attention to quantum information geometry [3], there is a standard affine connection $\nabla^{(m)}$, called the mixture connection (m-connection, for short), on a quantum state space $\mathcal{S}$. Since the m-connection stems from the natural convex structure of $\mathcal{S}$, the 2-tuple $\left(\mathcal{S}, \nabla^{(m)}\right)$ forms a flat manifold. It is then natural to inquire whether a Riemannian metric $g$ on the flat manifold $\left(\mathcal{S}, \nabla^{(m)}\right)$ is of Hessian type. A crucial answer to this problem was given by Nagaoka [3], who proved that a metric $g$ on $\left(\mathcal{S}, \nabla^{(m)}\right)$ induced from a generalized covariance is of Hessian type if and only if $g$ is the Bogoliubov metric. The canonical divergence of the corresponding dually flat structure is the Umegaki relative entropy [8].

A variety of other divergence-like quantities on a quantum state space $\mathcal{S}$ have been devised, and their relationships to information geometry have been discussed (see, for example, [915], and the references cited therein). In particular, it is known that every monotone quantum metric on $\mathcal{S}$ can be linked with some quantum relative entropy [12]. However, none of them except the Umegaki relative entropy is interpreted as the canonical divergence associated with some dually flat structure. In other words, we do not know any monotone quantum metric besides the Bogoliubov metric that admits a Hessian structure. It is then interesting to inquire whether there are other monotone metrics on a quantum state space that admit Hessian structures. The purpose of this paper is to give a positive answer to this question. The main result is the following

Theorem 2 Every monotone metric on a two-level quantum state space admits a Hessian structure.

The paper is organized as follows. In Sect. 2, we give a brief review of monotone metrics on a quantum state space $\mathcal{S}(\mathcal{H})$ over a finite-dimensional Hilbert space $\mathcal{H}$. In Sect. 3, we prove Theorem 2 by showing the existence of a local coordinate system $\left(\theta^{i}\right)$ and a function $\psi$ that satisfy (1) for any monotone metric on $\mathcal{S}\left(\mathbb{C}^{2}\right)$. In Sect. 4, we demonstrate the dually flat structures induced from the SLD and the RLD metrics in depth. Section 5 is devoted to concluding discussion.

\section{Monotone metrics on quantum state spaces}

Let $\mathcal{L}(\mathcal{H})$ and $\mathcal{L}_{\mathrm{sa}}(\mathcal{H})$ denote the sets of linear operators and selfadjoint operators on a finite-dimensional complex Hilbert space $\mathcal{H}$, and let $\mathcal{L}_{++}(\mathcal{H})$ denote the subset of $\mathcal{L}_{\text {sa }}(\mathcal{H})$ comprising strictly positive operators. In this paper, we are interested in information geometrical structures of the set

$$
\mathcal{S}:=\mathcal{S}(\mathcal{H}):=\left\{\rho \in \mathcal{L}_{++}(\mathcal{H}) \mid \operatorname{Tr} \rho=1\right\},
$$


which is called the quantum state space on $\mathcal{H}$.

In quantum information geometry, it is customary to use a pair of operator representations of tangent vectors called the m-representation and the e-representation [3]. The $m$ representation $X^{(m)}$ of a tangent vector $X \in T_{\rho} \mathcal{S}$ at $\rho \in \mathcal{S}$ is simply defined by

$$
X^{(m)}:=X \rho .
$$

As to the e-representation, there are several ways of introducing it. For later convenience, we adopt the following definition. Given a monotone increasing continuous function $f: \mathbb{R}_{++} \rightarrow$ $\mathbb{R}_{++}$satisfying the normalization $f(1)=1$ and the symmetry condition $f(t)=t f(1 / t)$, the e-representation $X_{f}^{(e)}$ of $X \in T_{\rho} \mathcal{S}$ associated with $f$ is defined by

$$
X_{f}^{(e)}:=f\left(\Delta_{\rho}\right)\left\{\rho^{-1}(X \rho)\right\},
$$

where $\Delta_{\rho}: \mathcal{L}(\mathcal{H}) \rightarrow \mathcal{L}(\mathcal{H})$ is the modular operator [16] for $\rho \in \mathcal{S}$ defined by

$$
\Delta_{\rho}: A \longmapsto \rho A \rho^{-1} .
$$

A Riemannian metric $g$ on $\mathcal{S}$ is then defined by the pairing

$$
g_{\rho}(X, Y)=\operatorname{Tr}\left\{X_{f}^{(e)} Y^{(m)}\right\}
$$

between e- and m-representations.

A metric $g$ is called monotone $[17,18]$ if it satisfies the monotonicity

$$
g_{\rho}(X, X) \geq g_{\gamma(\rho)}\left(\gamma_{*} X, \gamma_{*} X\right)
$$

for any completely positive trace-preserving map $\gamma: \mathcal{L}(\mathcal{H}) \rightarrow \mathcal{L}(\mathcal{H})$. The physical implication of this inequality is that the distinguishability of two nearby quantum states as measured by $g$ cannot be enhanced by any physical process $\gamma$. Thus, the monotonicity is regarded as a fundamental requirement for a metric. According to Petz's theorem [18], the metric $g$ given by (4) is monotone if and only if the representing function $f$ is operator monotone. In what follows, we denote the set of normalized symmetric operator monotone functions $f: \mathbb{R}_{++} \rightarrow \mathbb{R}_{++}$by $\mathcal{F}$. Typical examples are

$$
\frac{2 t}{1+t}, \quad \frac{t \log t}{t-1}, \quad \sqrt{t}, \quad \frac{t-1}{\log t}, \quad \frac{1+t}{2} .
$$

Note that $2 t /(1+t)$ and $(1+t) / 2$ are the minimum and the maximum elements of $\mathcal{F}$ [19]. In addition, $f \in \mathcal{F}$ if and only if $f^{\perp} \in \mathcal{F}$, where $f^{\perp}$ is the dual of $f$ defined by $f^{\perp}(t):=$ $t / f(t)$ [20]. Since the correspondence between $f$ and $f^{\perp}$ is one to one (in fact, $\left(f^{\perp}\right)^{\perp}=f$ ), we could express the e-representation (3) in terms of $f^{\perp} \in \mathcal{F}$ as follows:

$$
X_{f}^{(e)}=f^{\perp}\left(\Delta_{\rho}\right)^{-1}\left\{(X \rho) \rho^{-1}\right\} .
$$

Actually, in his original argument, Petz [18] employed this expression to represent monotone metrics.

Let us derive an explicit formula for the monotone metric $g$ associated with an operator monotone function $f \in \mathcal{F}$. Let

$$
\rho=\sum_{i=1}^{\operatorname{dim} \mathcal{H}} p_{i}\left|e_{i}\right\rangle\left\langle e_{i}\right|
$$


be a Schatten decomposition of $\rho \in \mathcal{S}$, where $\left\{e_{i}\right\}_{i}$ is an orthonormal basis of $\mathcal{H}$. Then, the e-representation $X_{f}^{(e)}$ associated with $f \in \mathcal{F}$ is given by

$$
X_{f}^{(e)}=\sum_{i, j} \frac{1}{p_{i}} f\left(\frac{p_{i}}{p_{j}}\right)\left|e_{i}\right\rangle\left\langle e_{i} \mid X^{(m)} e_{j}\right\rangle\left\langle e_{j}\right|,
$$

and the corresponding monotone metric $g$ is given by

$$
\begin{aligned}
g_{\rho}(X, Y) & =\sum_{i, j} \frac{1}{p_{i}} f\left(\frac{p_{i}}{p_{j}}\right)\left\langle e_{i} \mid X^{(m)} e_{j}\right\rangle\left\langle e_{j} \mid Y^{(m)} e_{i}\right\rangle \\
& =\sum_{i, j} \frac{1}{2}\left\{\frac{1}{p_{i}} f\left(\frac{p_{i}}{p_{j}}\right)+\frac{1}{p_{j}} f\left(\frac{p_{j}}{p_{i}}\right)\right\}\left\langle e_{i} \mid X^{(m)} e_{j}\right\rangle\left\langle e_{j} \mid Y^{(m)} e_{i}\right\rangle .
\end{aligned}
$$

Here, the second equality is due to the symmetry $f(t)=t f(1 / t)$. This formula will be used in calculating the components of a monotone metric $g$.

\section{Proof of Theorem 2}

In this section, we prove that any monotone metric on the quantum state space $\mathcal{S}\left(\mathbb{C}^{2}\right)$ admits a Hessian structure. Each element $\rho$ of $\mathcal{S}\left(\mathbb{C}^{2}\right)$ is uniquely specified by the so-called Stokes parameters $\left(x^{1}, x^{2}, x^{3}\right) \in \mathbb{R}^{3}$ as follows:

$$
\rho=\frac{1}{2}\left(I+x^{1} \sigma_{1}+x^{2} \sigma_{2}+x^{3} \sigma_{3}\right)=\frac{1}{2}\left[\begin{array}{cc}
1+x^{3} & x^{1}-\sqrt{-1} x^{2} \\
x^{1}+\sqrt{-1} x^{2} & 1-x^{3}
\end{array}\right],
$$

where $\sigma_{1}, \sigma_{2}, \sigma_{3}$ are the standard Pauli matrices. In order to assure the strict positivity of $\rho$, the parameters must belong to the open unit ball

$$
B=\left\{\left(x^{1}, x^{2}, x^{3}\right) \in \mathbb{R}^{3} \mid\left(x^{1}\right)^{2}+\left(x^{2}\right)^{2}+\left(x^{3}\right)^{2}<1\right\},
$$

which is sometimes referred to as the Bloch ball. In what follows, we adopt the Stokes parameters $\left(x^{1}, x^{2}, x^{3}\right)$ as a reference coordinate system of $\mathcal{S}\left(\mathbb{C}^{2}\right)$.

Due to the Codazzi equation (2), a Riemannian manifold $\left(\mathcal{S}\left(\mathbb{C}^{2}\right), g\right)$ admits a Hessian structure if and only if there is a local coordinate system $\theta=\left(\theta^{i}\right)$ that satisfies

$$
\frac{\partial g_{i j}}{\partial \theta^{k}}=\frac{\partial g_{k j}}{\partial \theta^{i}}
$$

or equivalently,

$$
\frac{\partial}{\partial \theta^{k}}\left(\frac{\partial x^{\lambda}}{\partial \theta^{i}} \frac{\partial x^{\mu}}{\partial \theta^{j}} g_{\lambda \mu}\right)=\frac{\partial}{\partial \theta^{i}}\left(\frac{\partial x^{\lambda}}{\partial \theta^{k}} \frac{\partial x^{\mu}}{\partial \theta^{j}} g_{\lambda \mu}\right)
$$

where

$$
g_{\lambda \mu}:=g\left(\frac{\partial}{\partial x^{\lambda}}, \frac{\partial}{\partial x^{\mu}}\right)
$$

are the components of $g$ with respect to the reference coordinate system $x=\left(x^{\lambda}\right)$, and Einstein's summation convention is used.

Suppose we are given an operator monotone function $f \in \mathcal{F}$. Then, by a direct computation using (5), the components $g_{\lambda \mu}$ of the corresponding monotone metric $g$ are given 
by

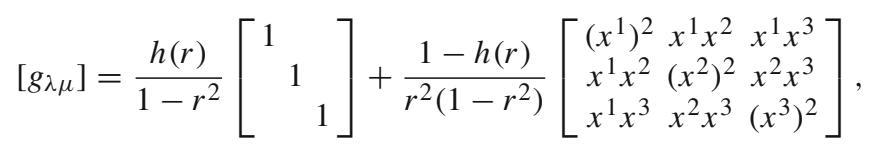

where $r:=\sqrt{\left(x^{1}\right)^{2}+\left(x^{2}\right)^{2}+\left(x^{3}\right)^{2}}$ and

$$
h(r):=\frac{1}{2}\left\{(1-r) f\left(\frac{1+r}{1-r}\right)+(1+r) f\left(\frac{1-r}{1+r}\right)\right\} .
$$

Note that $1-r^{2} \leq h(r) \leq 1$, which follows from $2 t /(1+t) \leq f(t) \leq(1+t) / 2$.

We shall prove Theorem 2 by showing the existence of a local coordinate system $\theta=\left(\theta^{i}\right)$ that satisfies the integrability conditions (7). To this end, we assume that the solution $x^{i}=$ $x^{i}(\theta)$ has the form

$$
x^{i}=F\left(e^{2 \theta^{1}}+e^{2 \theta^{2}}+e^{2 \theta^{3}}\right) e^{\theta^{i}}, \quad(i=1,2,3),
$$

where $F$ is a smooth positive function. This transformation will turn out to be pertinent to our problem because the functional form is covariant under the uniform shift of the origin $\theta^{i} \mapsto \theta^{i}+c$. Note that the local coordinate system $\theta=\left(\theta^{i}\right)$ in (9) only covers the positive octant of the Bloch ball $B$. This difficulty is evaded just by rotating the reference frame $\left(\sigma_{1}, \sigma_{2}, \sigma_{3}\right)$ in (6), which results in rotating the $x^{i}$-axes in $\mathbb{R}^{3}$. In this way, we can construct an atlas that covers the Bloch ball except the origin.

Since $\left(x^{1}\right)^{2}+\left(x^{2}\right)^{2}+\left(x^{3}\right)^{2}<1$, we have

$$
0<R F(R)^{2}<1,
$$

where $R:=e^{2 \theta^{1}}+e^{2 \theta^{2}}+e^{2 \theta^{3}}$. Further, since the Jacobian of the coordinate transformation (9) is

$$
\operatorname{det}\left[\frac{\partial x^{i}}{\partial \theta^{j}}\right]=e^{\theta^{1}+\theta^{2}+\theta^{3}} F(R)^{2}\left(2 R F^{\prime}(R)+F(R)\right),
$$

we claim that

$$
2 R F^{\prime}(R)+F(R) \neq 0 .
$$

Let us solve Eq. (7), which comprise nine partial differential equations corresponding to all combinations of $i, j, k \in\{1,2,3\}$ with $i<k$. It is shown by direct computations that if $i, j, k$ are different, the differences between both sides of (7) for the coordinate transformation (9) become zero; otherwise, the differences take the form

$$
\pm \frac{e^{2\left(\theta^{i}+\theta^{k}\right)}\left(2 R F^{\prime}(R)+F(R)\right)}{2 R\left(1-R F(R)^{2}\right)^{2}} \times H\left(R, F(R), F^{\prime}(R)\right),
$$

where $H\left(R, F(R), F^{\prime}(R)\right)$ is a certain function that is linear in $F^{\prime}(R)$. Therefore, under (10) and (11), the integrability conditions (7) are compressed into a single ordinary differential equation (ODE) $H\left(R, F(R), F^{\prime}(R)\right)=0$, which reads

$$
\begin{gathered}
F^{\prime}(R)=\frac{F(R)}{8 R\left(1-S^{2}\right)}\left[(2+S)(1-S)^{2} f\left(\frac{1+S}{1-S}\right)+(2-S)(1+S)^{2} f\left(\frac{1-S}{1+S}\right)\right. \\
\left.+2 S\left\{(1+S) f^{\prime}\left(\frac{1+S}{1-S}\right)-(1-S) f^{\prime}\left(\frac{1-S}{1+S}\right)\right\}-4\left(1-S^{2}\right)\right],
\end{gathered}
$$

where $S:=\sqrt{R F(R)^{2}}(=r)$. 
Since every operator monotone function $f$ is infinitely differentiable $[19,21]$, the righthand side of (12) is locally Lipschitz, and hence, the initial value problem for the ODE (12) has a unique solution on the domain (10). Moreover, any solution of (12) on the domain (10) satisfies (11). In fact, using the ODE (12) and the symmetry condition $f(t)=t f(1 / t)$ as well as its derivative, we have

$$
\begin{aligned}
& \frac{4\left(1-S^{2}\right)}{F(R)}\left(2 R F^{\prime}(R)+F(R)\right) \\
& =(2+S)(1-S)^{2} f\left(\frac{1+S}{1-S}\right)+(2-S)(1+S)^{2} f\left(\frac{1-S}{1+S}\right) \\
& \quad+2 S\left\{(1+S) f^{\prime}\left(\frac{1+S}{1-S}\right)-(1-S) f^{\prime}\left(\frac{1-S}{1+S}\right)\right\} \\
& =2(2+S)(1-S)^{2} f\left(\frac{1+S}{1-S}\right)+4 S(1+S) f^{\prime}\left(\frac{1+S}{1-S}\right),
\end{aligned}
$$

which is positive for $0<S<1$ because $f$ is positive and monotone increasing.

Let us choose a solution $F(R)$ of (12) arbitrarily. Then, the components $g_{i j}$ of $g$ with respect to the new local coordinate system $\theta=\left(\theta^{i}\right)$ are represented as follows (cf., Appendix A):

$$
\left[g_{i j}\right]=4 \phi_{1}(R)\left[\begin{array}{lll}
e^{2 \theta^{1}} & & \\
& e^{2 \theta^{2}} & \\
& & e^{2 \theta^{3}}
\end{array}\right]+4 \phi_{2}(R)\left[\begin{array}{ccc}
e^{4 \theta^{1}} & e^{2\left(\theta^{1}+\theta^{2}\right)} & e^{2\left(\theta^{1}+\theta^{3}\right)} \\
e^{2\left(\theta^{1}+\theta^{2}\right)} & e^{4 \theta^{2}} & e^{2\left(\theta^{2}+\theta^{3}\right)} \\
e^{2\left(\theta^{1}+\theta^{3}\right)} & e^{2\left(\theta^{2}+\theta^{3}\right)} & e^{4 \theta^{3}}
\end{array}\right],
$$

where

$$
\phi_{1}(R):=\frac{h(S)}{4\left(1-S^{2}\right)} F(R)^{2},
$$

and

$$
\phi_{2}(R):=\frac{1}{4 R\left(1-S^{2}\right)}\left[(1-h(S)) F(R)^{2}+4 R F(R) F^{\prime}(R)+4 R^{2} F^{\prime}(R)^{2}\right] .
$$

It is important to realize that

$$
\frac{d \phi_{1}(R)}{d R}=\phi_{2}(R)
$$

which is verified by a direct computation using the ODE (12). Thus, we conclude that the above $g_{i j}$ is rewritten as

$$
g_{i j}=\frac{\partial^{2}}{\partial \theta^{i} \partial \theta^{j}} \psi\left(e^{2 \theta^{1}}+e^{2 \theta^{2}}+e^{2 \theta^{3}}\right),
$$

where $\psi(R)$ is a primitive function of $\phi_{1}(R)$, i.e.,

$$
\psi(R):=\int^{R} \phi_{1}(s) \mathrm{d} s .
$$

This completes the proof of Theorem 2 .

Now, that we have found a potential function $\psi$ for $\theta$, computing the dual affine coordinate system $\eta=\left(\eta_{i}\right)$, the dual potential $\varphi(\eta)$ and the canonical divergence are standard. For 
example,

$$
\eta_{i}=\frac{\partial \psi}{\partial \theta^{i}}=2 e^{2 \theta^{i}} \phi_{1}\left(e^{2 \theta^{1}}+e^{2 \theta^{2}}+e^{2 \theta^{3}}\right) .
$$

In the next section, we demonstrate the dually flat structures induced from the SLD and the RLD metrics in more depth.

\section{Examples}

\subsection{SLD metric}

In quantum statistics, one of the most important Riemannian metrics on the quantum state space is the one derived from the symmetric logarithmic derivative (SLD, for short) [2224], and the corresponding metric is usually referred to as the SLD metric. The SLD metric corresponds to the operator monotone function $f(t)=2 t /(1+t)$, which is the minimum element of $\mathcal{F}$.

For this operator monotone function, the ODE (12) is reduced to

$$
F^{\prime}(R)=-\frac{F(R)^{3}}{2} \text {. }
$$

This ODE has the following solutions on the domain (10):

$$
F(R)=\frac{1}{\sqrt{R+C}}, \quad(C>0) .
$$

Note that changing the value of the integration constant $C$ yields shifting the origin of the $\theta$-coordinate system, and thus, the induced affine connection is invariant. Therefore, we set $C=1$ without loss of generality. In this case,

$$
\phi_{1}(R)=\frac{F(R)^{2}}{4}=\frac{1}{4(1+R)},
$$

and

$$
\psi(R)=\int_{0}^{R} \phi_{1}(s) \mathrm{d} s=\frac{1}{4} \log (1+R) .
$$

The dual affine coordinate system $\eta=\left(\eta_{i}\right)$ is

$$
\eta_{i}=\frac{\partial \psi(R)}{\partial \theta^{i}}=\frac{e^{2 \theta^{i}}}{2\left(1+e^{2 \theta^{1}}+e^{2 \theta^{2}}+e^{2 \theta^{3}}\right)},
$$

and the dual potential $\varphi(\eta)$ for the coordinate system $\eta$ is

$$
\begin{aligned}
\varphi(\eta):= & \max _{\theta}\left\{\theta^{i} \eta_{i}-\psi(\theta)\right\}=\frac{1}{4}\left[\sum_{i=1}^{3}\left(2 \eta_{i}\right) \log \left(2 \eta_{i}\right)\right. \\
& \left.+\left(1-\sum_{j=1}^{3}\left(2 \eta_{j}\right)\right) \log \left(1-\sum_{j=1}^{3}\left(2 \eta_{j}\right)\right)\right] .
\end{aligned}
$$


We thus arrive at the canonical divergence

$$
D(\rho \| \sigma):=\psi(\theta(\sigma))+\varphi(\eta(\rho))-\theta^{i}(\sigma) \eta_{i}(\rho)=\frac{1}{4} \sum_{i=0}^{3} p_{i} \log \frac{p_{i}}{q_{i}},
$$

where $\theta(\tau)=\left(\theta^{i}(\tau)\right)$ and $\eta(\tau)=\left(\eta_{i}(\tau)\right)$ denote the $\theta$ - and $\eta$-coordinates of $\tau \in \mathcal{S}\left(\mathbb{C}^{2}\right)$, and

$$
p_{i}:=\left\{\begin{array}{ll}
2 \eta_{i}(\rho), & (i=1,2,3) \\
1-\sum_{j=1}^{3} 2 \eta_{i}(\rho), & (i=0)
\end{array} \quad, \quad q_{i}=\left\{\begin{array}{ll}
2 \eta_{i}(\sigma), & (i=1,2,3) \\
1-\sum_{j=1}^{3} 2 \eta_{i}(\sigma), & (i=0)
\end{array} .\right.\right.
$$

To our surprise, the canonical divergence $D(\rho \| \sigma)$ is identical, up to the factor of $1 / 4$, to the classical Kullback-Leibler divergence between the probability distributions $p=$ $\left(p_{0}, p_{1}, p_{2}, p_{3}\right)$ and $q=\left(q_{0}, q_{1}, q_{2}, q_{3}\right)$, and the coordinate systems $\theta^{i}$ and $\eta_{i}$ correspond to the standard e- and $\mathrm{m}$-affine coordinate systems on the probability 3 -simplex in classical information geometry. It is interesting to realize that such a classical structure is hidden in the quantum state space $\mathcal{S}\left(\mathbb{C}^{2}\right)$ endowed with one of the most important quantum metrics, the SLD metric.

\subsection{RLD metric}

Another important Riemannian metric in quantum statistics is the one derived from the right logarithmic derivative (RLD, for short) [24,25], and the corresponding metric is usually referred to as the RLD metric. Since the RLD metric is a complex metric, we here take its real part and call it as the real RLD metric. The real RLD metric corresponds to the operator monotone function $f(t)=(1+t) / 2$, which is the maximum element of $\mathcal{F}$.

For this operator monotone function, the ODE (12) is reduced to

$$
F^{\prime}(R)=\frac{F(R)^{3}}{2\left(1-R F(R)^{2}\right)} .
$$

This ODE has the following implicit solutions:

$$
R F(R)^{2}-\log F(R)^{2}=C, \quad(C \in \mathbb{R}) .
$$

Set $C=0$ without loss of generality. Then, the solution on the domain (10) is explicitly written as

$$
F(R)=e^{-\frac{1}{2} W(-R)}, \quad\left(0<R<\frac{1}{e}\right)
$$

where $W$ is the Lambert $W$-function, i.e., the inverse function of $w \mapsto w e^{w}$ for $w \geq-1 / e$. For this solution,

$$
\phi_{1}(R)=\frac{F(R)^{2}}{4\left(1-R F(R)^{2}\right)}=\frac{1}{4\left(e^{W(-R)}-R\right)},
$$

and

$$
\psi(R)=\int_{0}^{R} \phi_{1}(s) \mathrm{d} s=-\frac{1}{4} W(-R) .
$$


The dual affine coordinate system $\eta=\left(\eta_{i}\right)$ is given by

$$
\eta_{i}=\frac{\partial \psi(R)}{\partial \theta^{i}}=\frac{e^{2 \theta^{i}}}{2\left(e^{W\left(-e^{2 \theta^{1}}-e^{2 \theta^{2}}-e^{2 \theta^{3}}\right)}-e^{2 \theta^{1}}-e^{2 \theta^{2}}-e^{2 \theta^{3}}\right)} .
$$

Unfortunately, the inverse transformation $\left(\eta_{i}\right) \mapsto\left(\theta^{i}\right)$ is obtained only implicitly, and thus, neither the dual potential $\varphi(\eta)$ nor the canonical divergence $D(\rho \| \sigma)$ can be written down in an explicit form.

\section{Discussion}

We have studied geometries of monotone quantum metrics and have found that any monotone metric on the quantum state space $\mathcal{S}\left(\mathbb{C}^{2}\right)$ admits a Hessian structure (Theorem 2). The key ingredient for the proof was the coordinate transformation of the form (9), which was valid for all monotone metrics. One may be aware that (9) does not induce the standard m-connection for the Bogoliubov metric because the local coordinate system $\theta=\left(\theta^{i}\right)$ only covers a single octant of the Bloch ball. In order to deal with this difficulty, we may use a generalized form of a coordinate transformation:

$$
x^{i}=F\left(G\left(\xi^{1}\right)^{2}+G\left(\xi^{2}\right)^{2}+G\left(\xi^{3}\right)^{2}\right) G\left(\xi^{i}\right), \quad(i=1,2,3) .
$$

For example, let us consider the case when $G(t):=t$, that is,

$$
x^{i}=F\left(\left(\xi^{1}\right)^{2}+\left(\xi^{2}\right)^{2}+\left(\xi^{3}\right)^{2}\right) \xi^{i}, \quad(i=1,2,3) .
$$

This form of transformation is covariant under the uniform scaling $\xi^{i} \mapsto c \xi^{i}$. With this transformation, the integrability conditions (7) for the Bogoliubov metric, which corresponds to the operator monotone function $f(t)=t \log t /(t-1)$, have solutions of the form

$$
F(R)=C \quad \text { and } \quad F(R)=\frac{1}{\sqrt{R}}\left(\frac{1-e^{-C \sqrt{R}}}{1+e^{-C \sqrt{R}}}\right),
$$

where $C$ is a positive constant. The first solution actually induces the standard m-connection for which the Stokes parameters $x=\left(x^{i}\right)$ form a global affine coordinate system. The second solution, on the other hand, induces another Hessian structure. To be more specific, letting $C=1$, the components $g_{i j}$ of the Bogoliubov metric with respect to the new coordinate system $\left(\xi^{i}\right)$ are shown to satisfy

$$
g_{i j}=\frac{\partial^{2}}{\partial \xi^{i} \partial \xi^{j}} \varphi\left(\left(\xi^{1}\right)^{2}+\left(\xi^{2}\right)^{2}+\left(\xi^{3}\right)^{2}\right),
$$

where

$$
\varphi(R):=\log \left(\cosh \frac{\sqrt{R}}{2}\right) .
$$

Unfortunately, the coordinate transformation (14) is not universal, in that there are monotone metrics, such as the SLD metric, for which the integrability conditions (7) do not have solutions on the Bloch ball. In this sense, the universality of the exponential-type transformation (9) is highly beneficial.

We have demonstrated rich geometrical structures of $\mathcal{S}\left(\mathbb{C}^{2}\right)$ induced from monotone metrics, and thus, Theorem 2 as well as its proof might be of some interest in itself. Such a view 
could be supported by the fact that the two-level quantum system is a building block in quantum information science. Nevertheless, the attainment here is far from satisfactory because our goal is to characterize all monotone metrics on a generic quantum state space $\mathcal{S}\left(\mathbb{C}^{d}\right)$ that admit Hessian structures. Unfortunately, the method presented here is not immediately applicable to a generic case because it essentially relies on a direct computation of the monotone metrics as well as the radial symmetric structure of the space $\mathcal{S}\left(\mathbb{C}^{2}\right)$ for which the coordinate transformation (9) is valid. In order to deal with a higher-dimensional quantum state space, some novel ideas are needed. In what follows, we make a preliminary consideration that may shed new light on this problem.

Let $\mathcal{M}$ be the totality of monotone metrics on $\mathcal{S}$. The set $\mathcal{M}$ is naturally regarded as a convex set, and the e-representation (3) of $X \in T_{\rho} \mathcal{S}$ establishes a one-to-one affine correspondence between $\mathcal{M}$ and $\mathcal{F}$. Further, due to Kubo and Ando's theory of operator means [20], each element of $\mathcal{F}$ is uniquely represented as

$$
f(t)=\int_{[0,1]} \frac{1+\lambda}{2}\left(\frac{t}{t+\lambda}+\frac{t}{t \lambda+1}\right) d \mu(\lambda),
$$

where $\mu$ is a probability measure on the closed interval $[0,1]$. This representation shows that the set $\mathcal{F}$ is a Choquet simplex, and its extreme points are given by

$$
f_{\lambda}(t)=\frac{1+\lambda}{2}\left(\frac{t}{t+\lambda}+\frac{t}{t \lambda+1}\right), \quad(\lambda \in[0,1]) .
$$

Specifically, the functions

$$
f_{1}(t)=\frac{2 t}{1+t} \quad \text { and } \quad f_{0}(t)=\frac{1+t}{2}
$$

correspond, respectively, to the SLD metric and the real RLD metric.

Now, in view of the decomposition (15), a possible approach to our problem may be decomposed into two questions as follows.

Problem 1 Do monotone metrics on $\mathcal{S}$ represented by the extreme points (16) of $\mathcal{F}$ admit Hessian structures?

Problem 2 Given two Riemannian metrics $g^{(0)}$ and $g^{(1)}$ on a manifold $M$, both of which admit Hessian structures, do their convex combinations $g^{(\alpha)}:=(1-\alpha) g^{(0)}+\alpha g^{(1)}$ also admit Hessian structures?

If both problems are resolved affirmatively, we can conclude that every monotone metric on a generic quantum state space $\mathcal{S}$ admits a Hessian structure. Conversely, if we find a counterexample to either of these problems, we will get a new perspective on the problem of flattenability of a Riemannian manifold. These questions are left for future research.

Acknowledgements The author is grateful to Shigeru Shuto and Daiji Tsutsui for fruitful discussions. The present study was supported by JSPS KAKENHI Grant No. JP17H02861.

Open Access This article is licensed under a Creative Commons Attribution 4.0 International License, which permits use, sharing, adaptation, distribution and reproduction in any medium or format, as long as you give appropriate credit to the original author(s) and the source, provide a link to the Creative Commons licence, and indicate if changes were made. The images or other third party material in this article are included in the article's Creative Commons licence, unless indicated otherwise in a credit line to the material. If material is not included in the article's Creative Commons licence and your intended use is not permitted by statutory regulation or exceeds the permitted use, you will need to obtain permission directly from the copyright holder. To view a copy of this licence, visit http://creativecommons.org/licenses/by/4.0/. 


\section{Appendix}

\section{A Proof of (13)}

Let us compute the components

$$
g_{i j}(\theta)=\left.\frac{\partial x^{\lambda}}{\partial \theta^{i}} \frac{\partial x^{\mu}}{\partial \theta^{j}} g_{\lambda \mu}(x)\right|_{x=x(\theta)} .
$$

For the coordinate transformation $x^{\lambda}=F(R) e^{\theta^{\lambda}}$ with $R=e^{2 \theta^{1}}+e^{2 \theta^{2}}+e^{2 \theta^{3}}$, we have

$$
\frac{\partial x^{\lambda}}{\partial \theta^{i}}=\left(F(R) \delta_{\lambda i}+F^{\prime}(R) 2 e^{2 \theta^{i}}\right) e^{\theta^{\lambda}} .
$$

On the other hand, it follows from (8) that the components $g_{\lambda \mu}(x(\theta))$ are written as

$$
\begin{aligned}
g_{\lambda \mu}(x(\theta)) & =\frac{h(S)}{1-S^{2}} \delta_{\lambda \mu}+\frac{1-h(S)}{S^{2}\left(1-S^{2}\right)} F(R)^{2} e^{\theta^{\lambda}} e^{\theta^{\mu}} \\
& =\frac{h(S)}{1-S^{2}} \delta_{\lambda \mu}+\frac{1-h(S)}{R\left(1-S^{2}\right)} e^{\theta^{\lambda}+\theta^{\mu}}
\end{aligned}
$$

where $S=\sqrt{R F(R)^{2}}$. Thus, a direct computation shows that

$$
\begin{aligned}
g_{i j}(\theta)= & \sum_{\lambda, \mu}\left(F(R) \delta_{\lambda i}+F^{\prime}(R) 2 e^{2 \theta^{i}}\right) e^{\theta^{\lambda}} \times\left(F(R) \delta_{\mu j}+F^{\prime}(R) 2 e^{2 \theta^{j}}\right) e^{\theta^{\mu}} \\
& \times\left(\frac{h(S)}{1-S^{2}} \delta_{\lambda \mu}+\frac{1-h(S)}{R\left(1-S^{2}\right)} e^{\theta^{\lambda}+\theta^{\mu}}\right) \\
= & \frac{h(S)}{1-S^{2}} F(R)^{2} e^{2 \theta^{i}} \delta_{i j} \\
& +\frac{1}{R\left(1-S^{2}\right)}\left[(1-h(S)) F(R)^{2}+4 R F(R) F^{\prime}(R)+4 R^{2} F^{\prime}(R)^{2}\right] e^{2\left(\theta^{i}+\theta^{j}\right)} .
\end{aligned}
$$

This proves (13).

\section{References}

1. H. Shima, The geometry of Hessian structures (World Scientific, Singapore, 2007)

2. S. Amari, Differential geometrical methods in statistics, vol. 28, Lecture notes in statistics (Springer, Berlin, 1985)

3. S. Amari, H. Nagaoka, Methods of information geometry (AMS/Oxford, Oxford, 2000)

4. H. Furuhata, H. Matsuzoe, H. Urakawa, Open problems in affine differential geometry and related topics. Interdiscip. Inf. Sci. 4, 125-127 (1999)

5. N. Ay, W. Tuschmann, Dually flat manifolds and global information geometry. Open Syst. Inf. Dyn. 9, 195-200 (2002)

6. R. Bryant, https://mathoverflow.net/questions/122308/, (2013)

7. S. Amari, J. Armstrong, Curvature of Hessian manifolds. Diff. Geom. Appl. 33, 1-12 (2014)

8. H. Umegaki, Conditional expectation in an operator algebra, IV (Entropy and information). Kodai Math. Sem. Rep. 14, 59-85 (1962)

9. D. Petz, Quasi-entropies for finite quantum systems. Rep. Math. Phys. 23, 57-65 (1986)

10. M. Ohya, D. Petz, Quantum entropy and its use (Springer, Berlin, 1993)

11. H. Hasegawa, D. Petz, Non-commutative extension of information geometry II, In: Quantum communication, computing, and measurement, Hirota et al. (eds.), 109-118 (Plenum, NY, 1997) 
12. A. Lesniewski, M.B. Ruskai, Monotone Riemannian metrics and relative entropy on noncommutative probability space. J. Math. Phys. 40, 5702-5724 (1999)

13. M. Müller-Lennert, F. Dupuis, O. Szehr, S. Fehr, M. Tomamichel, On quantum Rényi entropies: a new definition and some properties. J. Math. Phys. 54, 122203 (2013)

14. M.M. Wilde, A. Winter, D. Yang, Strong converse for the classical capacity of entanglement-breaking and Hadamard channels. Commun. Math. Phys. 331, 593-622 (2014)

15. K. Takahashi, A. Fujiwara, Information geometry of sandwiched Rényi $\alpha$-divergence. J. Phys. A Math. Theor. 50, 165301 (2017)

16. M. Takesaki, Tomita's theory of modular Hilbert algebras and its applications, vol. 128, Lecture notes in mathematics (Springer, Berlin, 1970)

17. E.A. Morozova, N.N. Chentsov, Markov invariant geometry on manifolds of states. J. Sov. Math. 56, 2648-2669 (1991). Original in Russian, 1989

18. D. Petz, Monotone metrics on matrix spaces. Linear Algebra Appl. 224, 81-96 (1996)

19. F. Hiai, D. Petz, Introduction to matrix analysis and applications (Hindustan/Springer, Berlin, 2014)

20. F. Kubo, T. Ando, Means of positive linear operators. Math. Ann. 246, 201-224 (1980)

21. R. Bhatia, Matrix analysis, graduate texts in mathematics 169 (Springer, NY, 1997)

22. C.W. Helstrom, Minimum mean-square error estimation in quantum statistics. Phys. Lett. 25A, 101-102 (1967)

23. C.W. Helstrom, Quantum detection and estimation theory (Academic Press, NY, 1976)

24. A.S. Holevo, Probabilistic and statistical aspects of quantum theory (North-Holland, Amsterdam, 1982)

25. H.P. Yuen, M. Lax, Multiple-parameter quantum estimation and measurement of non-selfadjoint observables. IEEE Trans. 19, 740-750 (1973) 